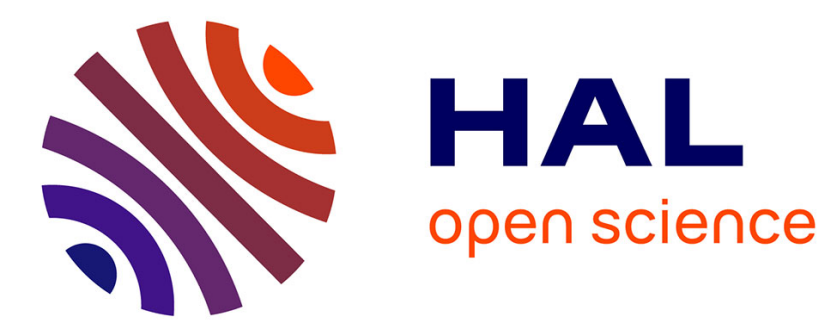

\title{
On Lisbon integrals
}

Daniel Barlet, Teresa Monteiro Fernandes

\section{To cite this version:}

Daniel Barlet, Teresa Monteiro Fernandes. On Lisbon integrals. Mathematische Zeitschrift, 2020, 10.1007/s00209-020-02540-6 . hal-02161740

\section{HAL Id: hal-02161740 https://hal.science/hal-02161740}

Submitted on 21 Jun 2019

HAL is a multi-disciplinary open access archive for the deposit and dissemination of scientific research documents, whether they are published or not. The documents may come from teaching and research institutions in France or abroad, or from public or private research centers.
L'archive ouverte pluridisciplinaire HAL, est destinée au dépôt et à la diffusion de documents scientifiques de niveau recherche, publiés ou non, émanant des établissements d'enseignement et de recherche français ou étrangers, des laboratoires publics ou privés. 


\title{
ON LISBON INTEGRALS
}

\section{DANIEL BARLET AND TERESA MONTEIRO FERNANDES}

\begin{abstract}
We introduce a family of integral transforms, the Lisbon Integrals, which naturally arise in the study of the affine space of unitary polynomials $P_{s}(z)$ where $s \in \mathbb{C}^{k}$ and $z \in \mathbb{C}, s_{i}$ identified to the $i$-th symmetric function of the roots of $P_{s}(z)$. We completely determine the $\mathcal{D}$-module (or system of partial differential equations) the Lisbon Integrals satisfy and prove that they are their unique global solutions of this $\mathcal{D}$-module.
\end{abstract}

\section{CONTEnTs}

1. Introduction 2

2. Lisbon Integrals and the differential system they satisfy 2

2.a. Lisbon integrals 2

2.b. The partial differential system 4

2.c. $\quad$ Example: The cases $k=2,3 \quad 8$

3. The left action of $\Gamma\left(\mathbb{C}, \mathcal{D}_{\mathbb{C}}\right)$ on Lisbon integrals 11

$\begin{array}{lll}4 . & \text { The } \mathcal{D}_{\mathbb{C}^{k}} \text {-module associated to Lisbon Integrals } & 12\end{array}$

$\begin{array}{lll}4 . a . & \text { An example } & 18\end{array}$

References 20

Date: $19 / 06 / 19$.

2010 Mathematics Subject Classification. 44A99, 32C35, 35A22, 35 A27, 58J15. 


\section{INTRODUCTION}

The purpose of this article is to show that the completely explicit differential system satisfied by the "Lisbon integrals" (defined in formulas (3) and (4) below) is in fact a $\mathcal{D}$-module obtained from a very simple one by the usual functorial operations on $\mathcal{D}$-modules (inverse image and direct image).

More precisely, the $\mathcal{D}$-module approach shows that the Lisbon integrals are holomorphic solutions of modules obtained as integral transforms associated to the affine space of unitary polynomials of degree $k$ in the variable $z \in \mathbb{C}$ with complex coefficients. Writing its elements in the form $P(s, z)=\sum_{i=0}^{k}(-1)^{i} s_{i} z^{k-i}$ with $s=\left(s_{1}, \cdots, s_{k}\right) \in \mathbb{C}^{k}$ with the convention $s_{0}=1$, this affine space (see [2, Ch. I]) is naturally identified to the hypersurface $H$ of $\mathbb{C}^{k+1}$ endowed with the coordinates $(s, z)$ of equation $s_{k}=(-1)^{k-1} \sum_{i=1}^{k}(-1)^{i} s_{i} z^{k-i}$. Let $j: H \subset \mathbb{C}^{k+1}$ denote the closed embedding and let $B_{H \mid \mathbb{C}^{k+1}}$ denote the regular holonomic $\mathcal{D}_{\mathbb{C}^{k+1}}$-module of holomorphic distributions supported by $H$. Since the restriction of the projection

$$
\pi: \mathbb{C}^{k+1} \longrightarrow \mathbb{C}^{k},(s, z) \mapsto s
$$

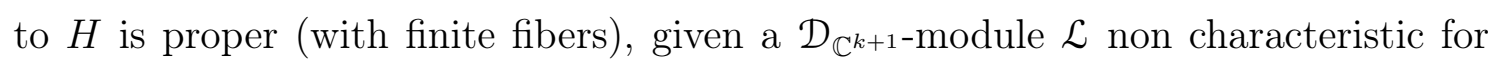
$H$, following [8] we obtain a complex in $\mathrm{D}_{\text {coh }}^{\mathrm{b}}\left(\mathcal{D}_{\mathbb{C}^{k}}\right)$, the integral transformed of $\mathcal{L}$, given by the composition of the usual derived functors of direct image and inverse image for $\mathcal{D}$-modules,

$$
D \pi_{*}\left(B_{H \mid \mathbb{C}^{k+1}} \stackrel{L}{\otimes}_{\mathcal{O}_{\mathbb{C}^{k+1}}} \mathcal{L}\right) \simeq D \pi_{*}\left(\mathcal{H}_{[H]}^{1}(\mathcal{L})\right) \simeq \pi_{*} j^{*} \mathcal{L}
$$

Note that $B_{H \mid \mathbb{C}^{k+1}}=\mathcal{H}_{[H]}^{1}\left(\mathcal{O}_{\mathbb{C}^{k+1}}\right)$ and that for such a module $\mathcal{L}$, we have, thanks to Kashiwara's equivalence theorem (cf Th 4.1 and Prop 4.2, [4]), $\mathcal{H}_{[H]}^{1}(\mathcal{L}) \simeq$ $\mathcal{H}^{0} D j_{*} D j^{*} \mathcal{L} \simeq j_{*} j^{*} \mathcal{L}$. In this note $\mathcal{L}$ is the quotient of $\mathcal{D}_{\mathbb{C}^{k+1}}$ by the ideal generated by the partial derivatives in $s_{i}, i=1, \cdots, k$, hence the sheaf of solutions of $\mathcal{L}$ is $p^{-1} \mathcal{O}_{\mathbb{C}}$ where $p(s, z)=z$.

As a consequence, we show that the Lisbon Integrals are exactly the global solutions of $\pi_{*} j^{*} \mathcal{L}$.

These computations illustrate the fact that it is not so easy, even in a rather simple situation, to follow explicitly the computations hidden in the "yoga" of $\mathcal{D}$-module theory.

\section{Lisbon Integrals AND THE DifFERENTIAL SYSTEM THEY SATISFY}

2.a. Lisbon integrals. For $\left(z_{1}, \ldots, z_{k}\right) \in \mathbb{C}^{k}$ denote $s_{1}, \ldots, s_{k}$ the elementary symmetric functions of $z_{1}, \ldots, z_{k}$. We shall consider in the sequel $s_{1}, \ldots, s_{k}$ as coordinates on $\mathbb{C}^{k} \simeq \mathbb{C}^{k} / \mathfrak{S}_{k}$, isomorphism given by the standard symmetric function theorem.

We shall denote $P_{s}(z):=\prod_{j=1}^{k}\left(z-z_{j}\right)=\sum_{h=0}^{k}(-1)^{h} s_{h} z^{k-h}$ with the convention $s_{0} \equiv 1$.

We shall often write $P(s, z)$ instead of $P_{s}(z)$ with no risk of ambiguity. The discriminant $\Delta(s)$ of $P_{s}$ is the polynomial in $s$ corresponding to the symmetric polynomial $\prod_{1 \leqslant i<j \leqslant k}\left(z_{i}-z_{j}\right)^{2}$ via the symmetric function theorem. 
Lemma 2.1. For $h \in \mathbb{N}$ and $f \in \mathcal{O}(\mathbb{C})$ any entire holomorphic function, let us define, for $R \gg\|s\|$,

$$
\varphi_{h}(s):=\frac{1}{2 i \pi} \int_{|\zeta|=R} \frac{f(\zeta) \zeta^{h} d \zeta}{P_{s}(\zeta)} .
$$

Then $\varphi_{h}(s)$ is independent of the choice of $R$ large enough and defines a holomorphic function on $\mathbb{C}^{k}$. For $\Delta(s) \neq 0$ we have

$$
\varphi_{h}(s)=\sum_{j=1}^{k} \frac{z_{j}^{h} f\left(z_{j}\right)}{P_{s}^{\prime}\left(z_{j}\right)}
$$

where $z_{1}, \ldots, z_{k}$ are the roots of $P_{s}(z)$.

Proof. The independence on $R$ large enough when $s$ stays in a compact set of $\mathbb{C}^{n}$ is clear. For $s$ in the interior of a compact set, $P_{s}(\zeta)$ does not vanish on $\{|\zeta|=R\}$ for $R$ large enough, so we obtain the holomorphy of $\varphi_{h}$ near any point in $\mathbb{C}^{k}$. The formula (2) is given by a direct application of the Residue's theorem.

In fact, it will be convenient to consider the $k$ functions $\varphi_{0}, \ldots, \varphi_{k-1}$ as the component of a vector valued function $\Phi:=\left(\begin{array}{c}\varphi_{0} \\ \varphi_{1} \\ \ldots \\ \varphi_{k-1}\end{array}\right)$. Defining $E(z):=\left(\begin{array}{c}1 \\ z \\ \ldots \\ z^{k-1}\end{array}\right)$ we obtain

$$
\Phi(s)=\frac{1}{2 i \pi} \int_{|\zeta|=R} \frac{f(\zeta) E(\zeta) d \zeta}{P_{s}(\zeta)}
$$

Definition 2.2. We call $\Phi$ (sometimes also denoted by $\Phi_{f}$ when precision is required) the Lisbon Integral associated to $f$

It will be also interesting to introduce another type of Lisbon integrals:

$$
\Psi(s):=\frac{1}{2 i \pi} \int_{|\zeta|=R} \frac{f(\zeta) E(\zeta) \cdot P_{s}^{\prime}(\zeta) d \zeta}{P_{s}(\zeta)}
$$

$\Psi$ will also be noted below by $\Psi_{f}$ when precision is required.

It is easy to see that this is again a vector valued holomorphic function on $\mathbb{C}^{k}$ and the Residue's theorem gives that, for $\Delta(s) \neq 0$, the component $\psi_{h}$ of $\Psi$ is given by:

$$
\psi_{h}(s)=\sum_{j=1}^{k} z_{j}^{h} f\left(z_{j}\right) .
$$

Proposition 2.3. If $f$ is not identically zero then $\Phi$ and $\Psi$ are non zero vectorvalued holomorphic functions on $\mathbb{C}^{k}$.

Proof. Suppose that $f$ is non identically zero. Then the statement follows as an immediate consequence of the non vanishing of the Van der Monde determinant of $z_{1}, \ldots, z_{k}$ when these complex numbers are pair-wise distinct. 
An example. Take $f \equiv 1$. Then formula (5) shows that $\psi_{h}(s)$ is the $h$-th Newton symmetric functions of the roots of the polynomial $P_{s}$. So it is a quasi-homogeneous polynomial in $s$ of weight $h$ (the weight of $s_{j}$ is $j$ by definition).

Let us show that we have $\varphi_{h}(s) \equiv 0$ for $h \in[0, k-2]$ and $\varphi_{k-1}(s) \equiv 1$ in this case. For $h \in[0, k-2]$ the formula (1) gives the estimate (with $f \equiv 1$ )

$$
\left|\varphi_{h}\right| \leqslant \frac{R^{h+1}}{(R-a)^{k-1}}
$$

if each root of $P_{s}$ is in the disc $\{|z| \leqslant a\}$ when $R>a>0$. When $R \rightarrow+\infty$ this gives $\varphi_{h}(s) \equiv 0$ for $h \in[0, k-2]$.

For $h=k-1$ write

$$
k z^{k-1}=P_{s}^{\prime}(z)-\sum_{h=1}^{k-1}(-1)^{h} k(k-h) s_{h} z^{k-h-1} .
$$

This gives, using the previous case and formula $(2)$, that $\varphi_{k-1}(s) \equiv 1$.

2.b. The partial differential system. Let us introduce the $(k, k)$ matrix $A$ associated to the polynomial $P_{s}$ by

$$
A:=\left(\begin{array}{cccccc}
0 & 1 & 0 & \cdots & & 0 \\
0 & 0 & 1 & 0 & \cdots & 0 \\
0 & & \cdots & & & 0 \\
& & & & & \\
0 & & \cdots & & 0 & 1 \\
(-1)^{k-1} . s_{k} & \cdots & (-1)^{h-1} . s_{h} & \cdots & \cdots & s_{1}
\end{array}\right)
$$

Theorem 2.b.1. The vector value holomorphic function $\Phi$ on $\mathbb{C}^{k}$ satisfies the following differential system

$$
(-1)^{k+h} \cdot \frac{\partial \Phi}{\partial s_{h}}(s)=\frac{\partial\left(A^{k-h} \Phi\right)}{\partial s_{k}}(s) \quad \forall s \in \mathbb{C}^{k} \quad \text { and } \quad \forall h \in[1, k-1] .
$$

Moreover, this system is integrabl $\rrbracket^{1}$ and if $\Phi$ is a solution of this system, so is $A \Phi$. The proof of this result will use several lemmas.

Lemma 2.4. Let $A$ be a $(k, k)$ matrix with entries in $\mathbb{C}[x]$ and put $B:=\lambda \frac{\partial A}{\partial x}$ where $\lambda$ is a complex number. Let $M$ be the $(2 k, 2 k)$ matrix given by

$$
M:=\left(\begin{array}{cc}
A & B \\
0 & A
\end{array}\right) .
$$

Then for each $p \in \mathbb{N}$ we have

$$
M^{p}=\left(\begin{array}{cc}
A^{p} & B_{p} \\
0 & A^{p}
\end{array}\right)
$$

\footnotetext{
${ }^{1}$ We shall explain in the proof what we mean here.
} 
where $B_{p}:=\lambda \frac{\partial\left(A^{p}\right)}{\partial x}$.

Proof. As the relation $\left(^{*}\right)$ is clear for $p=0,1$ let us assume that it has been proved for $p$ and let us prove it for $p+1$. We have:

$$
\left(\begin{array}{cc}
A^{p} & B_{p} \\
0 & A^{p}
\end{array}\right)\left(\begin{array}{ll}
A & B \\
0 & A
\end{array}\right)=\left(\begin{array}{cc}
A^{p+1} & A^{p} B+B_{p} A \\
0 & A^{p+1}
\end{array}\right)
$$

which allows to conclude.

Corollary 2.b.2. For each integer $p \in[0, k-1]$ the following equality holds in the module $\mathbb{C}^{k} \otimes_{\mathbb{C}} \mathbb{C}\left[s_{1}, \ldots, s_{k}, z\right] /\left(P^{2}\right)$ over the $\mathbb{C}$-algebra $\mathbb{C}\left[s_{1}, \ldots, s_{k}, z\right] /\left(P^{2}\right)$

$$
z^{p} E(z)=A^{p} E(z)+(-1)^{k-1} P_{s}(z) \frac{\partial\left(A^{p}\right)}{\partial s_{k}} E(z)
$$

In particular for any entire function on $z$,

$$
\Phi_{z f}=A(s) \Phi_{f}
$$

Moreover the following identity in the module $\mathbb{C}^{k} \otimes_{\mathbb{C}} \mathbb{C}\left[s_{1}, \ldots, s_{k}, z\right] /\left(P^{2}\right)$ holds

$$
P_{s}^{\prime}(z) E(z)=P_{s}^{\prime}(A) E(z)+(-1)^{k-1} P_{s}(z) \frac{\partial\left(P_{s}^{\prime}(A)\right)}{\partial s_{k}} E(z) .
$$

PROOF. In the basis $1, z, \cdots, z^{k-1}, P_{s}(z), z P_{s}(z), \cdots, z^{k-1} P_{s}(z)$ of this algebra which is a free rank $2 k$ module on $\mathbb{C}\left[s_{1}, \ldots, s_{k}\right]$, the multiplication by $z$ is given by the matrix $M$ of the previous lemma with $A$ as in (6) and with $B:=(-1)^{k-1} \frac{\partial A}{\partial s_{k}}$. This proves formula $(7)$.

As $P_{s}^{\prime}(z)=\sum_{h=0}^{k-1}(-1)^{h}(k-h) z^{k-h-1}$ does not depend on $s_{k}$ it is enough to sum up the previous equalities with $p=k-h-1$ with the convenient coefficients to obtain the equality $(7-$ bis $)$.

Lemma 2.5. Let $A$ be a $(k, k)$ matrix with entries in $\mathbb{C}[x, y]$. Assume that for some integer $q$ we have

then we have for any integer $h$

$$
\frac{\partial A}{\partial x} A^{q}=\frac{\partial A}{\partial y}
$$

$$
\frac{\partial A^{h}}{\partial x} A^{q}=\frac{\partial A^{h}}{\partial y}
$$

PROOF. The assertion is our hypothesis for $h=1$. Assume the assertion proved for some $h \geqslant 1$. Then Leibnitz rule gives:

$$
\frac{\partial A^{h+1}}{\partial x} \cdot A^{q}=\frac{\partial A^{h}}{\partial x} A^{q+1}+A^{h} \frac{\partial A}{\partial x} A^{q}
$$

which implies:

$$
\frac{\partial A^{h+1}}{\partial x} A^{q}=\left(\frac{\partial A^{h}}{\partial y} A+A^{h} \frac{\partial A}{\partial y}\right)=\frac{\partial A^{h+1}}{\partial y}
$$

which is our assertion for $h+1$. 
Corollary 2.b.3. For any $h \in[1, k]$ and any $p \in \mathbb{N}$ the matrix $A$ in (6) satisfies the relation:

$$
(-1)^{k-h} \frac{\partial A^{p}}{\partial s_{h}}=\frac{\partial A^{p}}{\partial s_{k}} A^{k-h}
$$

ProOf. The case $p=1$ of (7) is an easy direct computation on the matrix $A$. Assume that the assertion is proved for $p \geqslant 1$. Then Leibnitz's rule gives:

$$
\begin{gathered}
(-1)^{k-h} \frac{\partial A^{p+1}}{\partial s_{h}}=(-1)^{k-h} \frac{\partial A^{p}}{\partial s_{h}} A+A^{p}(-1)^{k-h} \frac{\partial A}{\partial s_{h}} \\
=\frac{\partial A^{p}}{\partial s_{k}} A^{k-h+1}+A^{p} \frac{\partial A}{\partial s_{k}} A^{k-h}=\frac{\partial A^{p+1}}{\partial s_{k}} A^{k-h}
\end{gathered}
$$

concluding the proof of (8).

Proof of The THEOREM 2.b.1. By derivation inside the integral in (3) we obtain:

$$
\begin{aligned}
& \frac{\partial \Phi}{\partial s_{h}}(s)=\frac{1}{2 i \pi} \int_{|\zeta|=R} f(\zeta) E(\zeta)(-1)^{h+1} \zeta^{k-h} \frac{d \zeta}{P_{s}(\zeta)^{2}} \text { and in particular } \\
& \frac{\partial \Phi}{\partial s_{k}}(s)=\frac{1}{2 i \pi} \int_{|\zeta|=R} f(\zeta) E(\zeta)(-1)^{k+1} \frac{d \zeta}{P_{s}(\zeta)^{2}}
\end{aligned}
$$

Now for $h \in[1, k-1]$ we use the formula of corollary 2.b.2 to obtain:

$$
\frac{\partial \Phi}{\partial s_{h}}=(-1)^{h+1} A^{k-h}(-1)^{k-1} \frac{\partial \Phi}{\partial s_{k}}+(-1)^{h+1}(-1)^{k-1} \frac{\partial A^{k-h}}{\partial s_{k}} \Phi
$$

that is to say:

$$
(-1)^{k+h} \frac{\partial \Phi}{\partial s_{h}}=\frac{\partial\left(A^{k-h} \Phi\right)}{\partial s_{k}} \quad \forall h \in[1, k]
$$

By the integrability of the system (@) we mean that for any $\Phi$ such that (@) holds, then the computation of the partial derivatives $\frac{\partial^{2} \Phi}{\partial s_{h} \partial s_{j}}$ using the system (@) gives a symmetric result in $(h, j)$ for any pair $(h, j)$ in $[1, k]$. Note that if $h$ or $j$ is equal to $k$ the assertion is trivial.

So consider a couple $(h, j) \in[1, k-1]^{2}$. Thanks to corollary 2.b.3 we have :

$$
\begin{aligned}
(-1)^{h+j} & \cdot \frac{\partial^{2} \Phi}{\partial s_{j} \partial s_{h}}=(-1)^{k-j} \frac{\partial}{\partial s_{k}}\left[\frac{\partial\left(A^{k-h} \Phi\right)}{\partial s_{j}}\right] \\
& =(-1)^{k-j} \frac{\partial}{\partial s_{k}}\left[\frac{\partial A^{k-h}}{\partial s_{j}} \Phi+A^{k-h} \frac{\partial \Phi}{\partial s_{j}}\right] \\
& =\frac{\partial}{\partial s_{k}}\left[\frac{\partial A^{k-h}}{\partial s_{j}} A^{k-j} \Phi+A^{k-h} \frac{\partial\left(A^{k-j} \Phi\right)}{\partial s_{k}}\right] \\
& =\frac{\partial^{2}}{\partial s_{k}^{2}}\left[A^{2 k-h-j} \Phi\right]
\end{aligned}
$$

which is symmetric in $(h, j)$.

To finish the proof of the theorem we have to show that $A \Phi$ is a solution of (@) when $\Phi$ is a solution of (@). This is given by the following computation 


$$
\begin{array}{r}
(-1)^{k-h} \frac{\partial(A \Phi)}{\partial s_{h}}=(-1)^{k-h} \frac{\partial A}{\partial s_{h}} \Phi+(-1)^{k-h} A \frac{\partial \Phi}{\partial s_{h}} \\
=\frac{\partial A}{\partial s_{k}} \cdot A^{k-h} \Phi+A \frac{\partial A^{k-h} \Phi}{\partial s_{k}}=\frac{\partial\left(A^{k-h}(A \Phi)\right)}{\partial s_{k}}
\end{array}
$$

which also uses corollary 2.b.3.

REMARK. A consequence of our computation on the integrability of the system (@) is the fact that for any solution $\Phi$ and any pair $(h, j) \in[1, k]$ the second order partial derivative $\frac{\partial^{2} \Phi}{\partial s_{j} \partial s_{h}}$ only depends on $h+j$. This implies that any scalar Lisbon integral $\varphi_{h}$ satisfies

$$
\frac{\partial^{2} \varphi_{h}}{\partial s_{p} \partial s_{q+1}}=\frac{\partial^{2} \varphi_{h}}{\partial s_{p+1} \partial s_{q}} \quad \forall p, q \text { such that } 1 \leqslant p<q \leqslant k-1
$$

The following corollary of theorem 2.b.1 describes the analogous system for the the vector functions $\Psi$ defined in (4) which is singular along the discriminant subset $\{\Delta(s)=0\}$ in $\mathbb{C}^{k}$.

Corollary 2.b.4. The vector value holomorphic function $\Psi$ on $\mathbb{C}^{k}$ satisfies the following differential system:

$$
(-1)^{k+h} \frac{\partial \Psi}{\partial s_{h}}(s)=\frac{\partial\left(A^{k-h} \Psi\right)}{\partial s_{k}}(s)+(-1)^{k}(k-h) A^{k-h-1} P_{s}^{\prime}(A)^{-1} \Psi(s)
$$

for all $h \in[1, k-1]$, which is singular along the discriminant sub-set

$$
\Delta:=\left\{s \in \mathbb{C}^{k} / \Delta(s)=0\right\} .
$$

Again, if $\Psi$ is any solution of (@@) then $A \Psi$ is also a solution of (@@).

Proof. Define $F(z):=P^{\prime}(z) E(z)$. Then using formula $(7)$ we obtain $\Psi(s)=$ $P_{s}^{\prime}(A) \Phi(s)$ and this gives for each $h \in[1, k-1]$

$$
(-1)^{k-h} \frac{\partial \Psi}{\partial s_{h}}(s)=(-1)^{k-h} A^{k-h-1} \Phi(s)+\sum_{p=0}^{k-1}(-1)^{p}(k-p)(-1)^{k-h} s_{p} \cdot \frac{\partial\left(A^{k-p-1} \Phi\right)}{\partial s_{h}}(s)
$$

and using now the fact that $A^{k-p-1} \Phi$ is solution of (@) we obtain

$$
\begin{aligned}
(-1)^{k-h} \frac{\partial \Psi}{\partial s_{h}}(s) & =(-1)^{k-h} A^{k-h-1} \Phi(s)+\sum_{p=0}^{k-1}(-1)^{p}(k-p) s_{p} \cdot \frac{\partial\left(A^{2 k-p-h-1} . \Phi\right)}{\partial s_{k}}(s) \\
(-1)^{k-h} \frac{\partial \Psi}{\partial s_{h}}(s) & =(-1)^{k-h} A^{k-h-1} \Phi(s)+\frac{\partial\left(A^{k-h} P_{s}^{\prime}(A) \Phi\right)}{\partial s_{k}}(s) \\
(-1)^{k-h} \frac{\partial \Psi}{\partial s_{h}}(s) & =(-1)^{k-h} A^{k-h-1} P_{s}^{\prime}(A)^{-1} \Psi(s)+\frac{\partial\left(A^{k-h} \Psi\right)}{\partial s_{k}}(s)
\end{aligned}
$$

Since $P_{s}^{\prime}(A)$ commutes with $A$, the last assertion is easy. 
2.c. Example: The cases $k=2,3$. The left ideal of partial differential operators in the Weyl algebra $\mathbb{C}\left[s_{1}, \ldots, s_{k}\right]\left\langle\partial_{s_{1}}, \ldots, \partial_{s_{k}}\right\rangle$ which anihilate Lisbon integrals is described in the article [1].

Below we will explicit the cases $k=2$ and $k=3$.

Case $k=2$ :

Here we use the notations $s:=s_{1}$ and $p:=s_{2}$. In that case, the differential system (@) becomes:

$$
\begin{aligned}
& -\frac{\partial \Phi_{0}}{\partial s}=\frac{\partial \Phi_{1}}{\partial p} \\
& -\frac{\partial \Phi_{1}}{\partial s}=-\Phi_{0}-p \frac{\partial \Phi_{0}}{\partial p}+s \frac{\partial \Phi_{1}}{\partial p}
\end{aligned}
$$

corresponding to the matrix $A:=\left(\begin{array}{cc}0 & 1 \\ -p & s\end{array}\right)$. Differentiating (9) with respect to $p$ we obtain, after substituting via (10)

$$
\begin{aligned}
& \frac{\partial^{2} \Phi_{0}}{\partial s^{2}}=-2 \frac{\partial \Phi_{0}}{\partial p}-p \frac{\partial^{2} \Phi_{0}}{\partial p^{2}}-s \frac{\partial^{2} \Phi_{0}}{\partial s \partial p} \\
& \text { and so } \quad \frac{\partial^{2} \Phi_{0}}{\partial s^{2}}+s \frac{\partial^{2} \Phi_{0}}{\partial s \partial p}+p \frac{\partial^{2} \Phi_{0}}{\partial p^{2}}+2 \frac{\partial \Phi_{0}}{\partial p}=0
\end{aligned}
$$

Differentiating (10) with respect to $s$ we obtain, after substituting via (9)

$$
\begin{aligned}
& -\frac{\partial^{2} \Phi_{1}}{\partial s^{2}}=\frac{\partial \Phi_{1}}{\partial p}+p \frac{\partial^{2} \Phi_{1}}{\partial p^{2}}+\frac{\partial \Phi_{1}}{\partial p}+s \frac{\partial^{2} \Phi_{1}}{\partial s \partial p} \\
& \text { and so } \quad \frac{\partial^{2} \Phi_{1}}{\partial s^{2}}+s \frac{\partial^{2} \Phi_{1}}{\partial s \partial p}+p \frac{\partial^{2} \Phi_{1}}{\partial p^{2}}+2 \frac{\partial \Phi_{1}}{\partial p}=0
\end{aligned}
$$

Then the second order differential operator of weight -2

$$
\Theta:=\frac{\partial^{2}}{\partial s^{2}}+s \frac{\partial^{2}}{\partial s \partial p}+p \frac{\partial^{2}}{\partial p^{2}}+2 \frac{\partial}{\partial p}
$$

anihilates $\Phi_{0}$ and $\Phi_{1}$ for any solution $\Phi$ of the system (10), (11).

A DiReCT PRoOf THAT $\Theta$ ANihilates $\varphi_{m}$ FOR ALL $m \in \mathbb{N}$. We have, for any entire function $f: \mathbb{C} \rightarrow \mathbb{C}$ and for $R \gg \max \{|s|,|p|\}$ :

$$
\varphi_{m}(s, p)=\frac{1}{2 i \pi} \int_{|\zeta|=R} f(\zeta) \frac{\zeta^{m} d \zeta}{\zeta^{2}-s \zeta+p}
$$


This gives:

$$
\begin{aligned}
& \frac{\partial \varphi_{m}}{\partial s}(s, p)=\frac{1}{2 i \pi} \int_{|\zeta|=R} f(\zeta) \frac{\zeta^{m+1} d \zeta}{\left(\zeta^{2}-s \zeta+p\right)^{2}} \\
& \frac{\partial \varphi_{m}}{\partial p}(s, p)=-\frac{1}{2 i \pi} \int_{|\zeta|=R} f(\zeta) \frac{\zeta^{m} d \zeta}{\left(\zeta^{2}-s \zeta+p\right)^{2}} \\
& \frac{\partial^{2} \varphi_{m}}{\partial s^{2}}(s, p)=2 \frac{1}{2 i \pi} \int_{|\zeta|=R} f(\zeta) \frac{\zeta^{m+2} d \zeta}{\left(\zeta^{2}-s \zeta+p\right)^{3}} \\
& \frac{\partial^{2} \varphi_{m}}{\partial s \partial p}(s, p)=-2 \frac{1}{2 i \pi} \int_{|\zeta|=R} f(\zeta) \frac{\zeta^{m+1} d \zeta}{\left(\zeta^{2}-s \zeta+p\right)^{3}} \\
& \frac{\partial^{2} \varphi_{m}}{\partial p^{2}}(s, p)=2 \frac{1}{2 i \pi} \int_{|\zeta|=R} f(\zeta) \frac{\zeta^{m} d \zeta}{\left(\zeta^{2}-s \zeta+p\right)^{3}}
\end{aligned}
$$

Now it is easy to check that $(d)+s(e)+p(f)+2(c)=0$.

Case $k=3$ :

The differential system system (@) becomes now:

$$
(-1)^{k-h} \partial_{h} \Phi=\partial_{k}\left(A^{k-h} \Phi\right) \quad \text { for } \quad h \in[1, k-1]
$$

with $k=3$. Here $\Phi=\left(\begin{array}{c}\Phi_{0} \\ \Phi_{1} \\ \Phi_{2}\end{array}\right)$ and $A=\left(\begin{array}{ccc}0 & 1 & 0 \\ 0 & 0 & 1 \\ s_{3} & -s_{2} & s_{1}\end{array}\right)$. Explicitely this gives:

$$
\begin{aligned}
& \partial_{1} \Phi_{0}=\partial_{3} \Phi_{2} \\
& \partial_{1} \Phi_{1}=\partial_{3}\left(s_{3} . \Phi_{0}-s_{2} \Phi_{1}+s_{1} \Phi_{2}\right) \\
& \partial_{1} \Phi_{2}=\partial_{3}\left(s_{1} s_{3} \Phi_{0}-\left(s_{3}-s_{1} s_{2}\right) \Phi_{1}+\left(s_{1}^{2}-s_{2}\right) \Phi_{2}\right) \\
& -\partial_{2} \Phi_{0}=\partial_{3} \Phi_{1} \\
& -\partial_{2} \Phi_{1}=\partial_{3} \Phi_{2} \\
& -\partial_{2} \Phi_{2}=\partial_{3}\left(s_{3} \Phi_{0}-s_{2} \Phi_{1}+s_{1} \Phi_{2}\right)
\end{aligned}
$$

which implies the equalities

$$
\partial_{1} \Phi_{0}=-\partial_{2} \Phi_{1}=\partial_{3} \Phi_{2} \quad \partial_{1} \Phi_{1}=-\partial_{2} \Phi_{2}
$$

Then we deduce that:

$$
\left(\partial_{2}^{2}-\partial_{1} \partial_{3}\right)\left(\Phi_{j}\right)=0 \quad \text { for } \quad j=0,1,2
$$

because

$$
\begin{gathered}
\partial_{2}^{2} \Phi_{0}=-\partial_{2} \partial_{3} \Phi_{1}=\partial_{3} \partial_{3} \Phi_{2}=\partial_{3} \partial_{1} \Phi_{0} \\
\partial_{1} \partial_{3} \Phi_{1}=-\partial_{1} \partial_{2} \Phi_{0}=\partial_{2} \partial_{3} \Phi_{2}=\partial_{2}^{2} \Phi_{1} \\
\text { and } \partial_{2}^{2} \Phi_{2}=-\partial_{2} \partial_{1} \Phi_{1}=\partial_{3} \partial_{2} \Phi_{2}
\end{gathered}
$$


Lemma 2.6. Let us define:

$$
\begin{aligned}
& \mathcal{L}:=\partial_{1}^{2}+s_{1} \partial_{1} \partial_{2}+s_{2} \partial_{2}^{2}+s_{3} \partial_{2} \partial_{3}+2 \partial_{2} \\
& \mathcal{L}^{\prime}:=\partial_{1} \partial_{2}+s_{1} \partial_{2}^{2}+s_{2} \partial_{2} \partial_{3}+s_{3} \partial_{3}^{2}+2 \partial_{3}
\end{aligned}
$$

Then we have:

$$
\mathcal{L} \Phi_{0}=0 \quad \mathcal{L}^{\prime} \Phi_{1}=0 \quad \text { et } \quad \mathcal{L} \Phi_{2}=0
$$

and also:

$$
\partial_{3} \mathcal{L}-\partial_{2} \mathcal{L}^{\prime} \in \mathcal{D}_{\mathbb{C}^{k}}\left(\partial_{1} \partial_{3}-\partial_{2}^{2}\right)
$$

This entails that $\partial_{3} \mathcal{L}$ and $\partial_{2} \mathcal{L}^{\prime}$ anihilate $\Phi_{j}$ for $j=0,1,2$.

Proof. We have

$$
\begin{aligned}
& \begin{array}{l}
\partial_{1} \Phi_{1}=\Phi_{0}+s_{3} . \partial_{3} \Phi_{0}-s_{2} . \partial_{3} \Phi_{1}+s_{1} \cdot \partial_{3} \Phi_{2} \\
\quad=\Phi_{0}+s_{3} \cdot \partial_{3} \Phi_{0}+s_{2} . \partial_{2} \Phi_{0}+s_{1} \cdot \partial_{1} \Phi_{0}
\end{array} \\
& \text { and so } \quad \partial_{2} \partial_{1} \Phi_{1}=-\partial_{1}^{2} \Phi_{0}= \\
& \partial_{2} \Phi_{0}+s_{3} . \partial_{2} \partial_{3} \Phi_{0}+\partial_{2} \Phi_{0}+s_{2} . \partial_{2}^{2} \Phi_{0}+s_{1} . \partial_{1} \partial_{2} \Phi_{0}
\end{aligned}
$$

and so $\Phi_{0}$ is killed by $\mathcal{L}$ as $\left(\partial_{1} \partial_{3}-\partial_{2}^{2}\right) \Phi_{0}=0$.

In a similar way:

$$
\begin{aligned}
& \partial_{1} \Phi_{1}=-\partial_{2} \Phi_{2}=\partial_{3}\left(s_{3} . \Phi_{0}-s_{2} . \Phi_{1}+s_{1} . \Phi_{2}\right) \\
& \begin{aligned}
\partial_{2} \partial_{1} \Phi_{1} & =-\partial_{3}\left(s_{3} . \partial_{3} \Phi_{1}\right)-\partial_{2} \partial_{3}\left(s_{2} . \Phi_{1}\right)-s_{1} \cdot \partial_{2}^{2} \Phi_{1} \\
& =-\partial_{3} \Phi_{1}-s_{3} . \partial_{3}^{2} \Phi_{1}-\partial_{3} \Phi_{1}-s_{2} . \partial_{2} \partial_{3} \Phi_{1}-s_{1} . \partial_{2}^{2} \Phi_{1}
\end{aligned}
\end{aligned}
$$

showing that $\mathcal{L}^{\prime}$ anihilates $\Phi_{1}$.

In a similar way:

$$
\begin{aligned}
\partial_{1} \Phi_{2} & =s_{1} \cdot \Phi_{0}+s_{1} \cdot s_{3} \partial_{3} \Phi_{0}+\Phi_{1}+\left(s_{3}-s_{1} \cdot s_{2}\right) \cdot \partial_{3} \Phi_{1}+\left(s_{1}^{2}-s_{2}\right) \partial_{3} \Phi_{2} \\
& =s_{1} \cdot \partial_{1} \Phi_{1}+\Phi_{1}-s_{2} \cdot \partial_{3} \cdot \Phi_{2}+s_{3} \cdot \partial_{3} \Phi_{1} \\
& =s_{1} \cdot \partial_{1} \Phi_{1}+\Phi_{1}+s_{2} \cdot \partial_{2} \cdot \Phi_{1}+s_{3} . \partial_{3} \Phi_{1} \\
\partial_{1}^{2} \Phi_{2} & =-\partial_{2} \Phi_{2}-s_{1} \cdot \partial_{1} \partial_{2} \Phi_{2}-\partial_{2} \Phi_{2}+s_{2} \cdot \partial_{2}\left(-\partial_{2} \Phi_{2}\right)+s_{3} . \partial_{3} \cdot\left(\partial_{1} \Phi_{1}\right) \\
& =-2 \partial_{2} \Phi_{2}-s_{1} \cdot \partial_{1} \partial_{2} \Phi_{2}-s_{2} \cdot \partial_{2}^{2} \Phi_{2}-s_{3} \cdot \partial_{2} \partial_{3} \Phi_{2}
\end{aligned}
$$

and $\Phi_{2}$ is anihilated by $\mathcal{L}$.

Let us prove now the relation $(r)$ :

$$
\begin{aligned}
& \partial_{3} \mathcal{L}=\partial_{3} \partial_{1}^{2}+s_{1} \cdot \partial_{1} \partial_{2} \partial_{3}+s_{2} . \partial_{1} \partial_{3}^{2}+s_{3} \cdot \partial_{2} \partial_{3}^{2}+3 \partial_{2} \partial_{3} \\
& \partial_{2} \mathcal{L}^{\prime}=\partial_{1} \partial_{2}^{2}+s_{3} \cdot \partial_{2} \cdot \partial_{3}^{2}+s_{2} \cdot \partial_{2}^{2} \partial_{3}+s_{1} \cdot \partial_{2}^{3}+3 \partial_{2} \partial_{3}
\end{aligned}
$$

this allows to conclude, as

$$
\begin{aligned}
& \partial_{3} \partial_{1}^{2}-\partial_{1} \partial_{2}^{2}=\partial_{1}\left(\partial_{1} \partial_{3}-\partial_{2}^{2}\right) \\
& \partial_{1} \partial_{2} \partial_{3}-\partial_{2}^{3}=\partial_{2}\left(\partial_{1} \partial_{3}-\partial_{2}^{2}\right)
\end{aligned}
$$

For the study of differential operators anihilating all the scalar components of the Lisbon integrals (for any value of $k$ ) see [1]. 


\section{The left action of $\Gamma\left(\mathbb{C}, \mathcal{D}_{\mathbb{C}}\right)$ On Lisbon integrals}

For each entire function $f$ of the variable $z$ we shall henceforward denote by $\Phi_{f}$ the associated Lisbon integral (previously generically denoted by $\Phi$ ). Clearly the assignement

$$
f \mapsto \Phi_{f}
$$

is $\mathbb{C}$-linear and, according to Proposition 2.3, it is injective.

Lemma 3.1. Let $f$ be an entire function on $\mathbb{C}$ and let $\Phi_{f}$ the corresponding Lisbon integral.

(1) Let $h$ be an entire function of $z$. Then $\Phi_{h f}=h(A(s)) \Phi_{f}$. In particular $\Phi_{f}=f(A(s)) \Phi_{1}$.

(2) We have the identity

$$
\Phi_{\partial_{z}(f)}(s)=-\nabla \Phi_{f}(s)+\left(\sum_{h=0}^{k-1}(k-h) s_{h} \partial_{s_{h+1}}\right)\left(\Phi_{f}\right)(s)
$$

where $\nabla$ is the constant $(k, k)$ matrix $\left(\begin{array}{ccccc}0 & 0 & 0 & \ldots & 0 \\ 1 & 0 & 0 & \ldots & 0 \\ 0 & 2 & 0 & \ldots & 0 \\ 0 & 0 & \ldots & 0 \\ 0 & \ldots & 0 & k-1 & 0\end{array}\right)$.

Proof. When $h$ is a polynomial on $z$, statement (1) follows easily in Corollary 2.b.2. For an arbitrary entire function $h$, it is a consequence of [2, Lem 3.1.8].

Let us prove (2): Consider the Lisbon integral

$$
\Phi_{\partial_{z} f}(s)=\frac{1}{2 i \pi} \int_{|\zeta|=R} f^{\prime}(\zeta) E(\zeta) \frac{d \zeta}{P_{s}(\zeta)}
$$

Integration by parts gives, as $\partial_{z}(E)(z)=\nabla E(z)$ :

$$
\Phi_{\partial_{z}(f)}(s)=-\nabla \phi_{f}(s)+\frac{1}{2 i \pi} \int_{|\zeta|=R} f(\zeta) E(\zeta) \frac{P_{s}^{\prime}(\zeta)}{P_{s}(\zeta)^{2}} d \zeta
$$

Now, using the equalities $P_{s}^{\prime}(\zeta)=\sum_{h=0}^{k-1}(-1)^{h}(k-h) s_{h} \zeta^{k-h-1}$ and

$$
\frac{\partial \Phi_{f}}{\partial s_{h}}(s)=-\int_{|\zeta|=R} f(\zeta) E(\zeta) \frac{(-1)^{h} \zeta^{k-h} d \zeta}{P_{s}(\zeta)^{2}}
$$

we obtain the formula of the lemma.

REMARK. From the formula $(*)$ and using the fact that $P_{s}^{\prime}(\zeta) E(\zeta)=P_{s}^{\prime}(A) E(\zeta)$ we obtain also the formula

$$
\Phi_{\partial_{z}(f)}(s)=-\nabla \Phi_{f}(s)+(-1)^{k} \frac{\partial\left(P_{s}^{\prime}(A) \Phi_{f}\right)}{\partial_{s_{k}}}(s)
$$

It is not obvious that when $\Phi$ is solution of the system (@), then $\left(\Phi \partial_{z}\right)(s):=$ $-\nabla \Phi(s)+(-1)^{k} \frac{\partial\left(P_{s}^{\prime}(A) \Phi\right)}{\partial_{s_{k}}}(s)$ (given by the formula $\left({ }^{* *}\right)$ ) is also a solution of the same system. A direct verification of this fact is consequence of the formula given in the lemma below. 
In order to verify that we have $\Phi_{(z . f)^{\prime}}=\Phi_{f}+\Phi_{z f^{\prime}}$ corresponding to the usual relation $\partial_{z} z-z \partial_{z}=1$ the following lemma is useful.

Lemma 3.2. We have the following identity:

$$
\nabla A-A \nabla+I d=(-1)^{k-1} \partial_{k}(A) P_{s}^{\prime}(A), \quad \forall s \in \mathbb{C}^{k} .
$$

ProOF. It is an easy computation to obtain that $\nabla A-A \nabla+I d$ is the matrix which have all lines equal to 0 excepted its last one which is given by $\left(x_{1}, \ldots, x_{k}\right)$ with $x_{h}=(-1)^{k-h} h s_{k-h}$ for $h \in[1, k]$ with $s_{0} \equiv 0$. On the other hand, the matrix $(-1)^{k-1} \partial_{k} A$ has only a non zero term at the place $(k, 1)$ which equal to 1 , so it is quite easy to see that $(-1)^{k-1}\left(\partial_{k} A\right) A^{p}$ has only a non zero term at the place $(k, p+1)$ with value $(-1)^{k-1}$. According to the computation of $(-1)^{k-1} \partial_{k}(A) P_{s}^{\prime}(A)$ we conclude the desired formula.

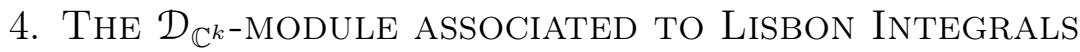

We shall begin by recalling some basic facts on $\mathcal{D}$-modules and fixing our notations.

For a morphism of manifolds $f: Y \rightarrow X$, we use the notation of [7]

$$
f_{d}:={ }^{t} f^{\prime}: T^{*} X \times_{X} \times Y \longrightarrow T^{*} Y
$$

and

$$
f_{\pi}: T^{*} X \times_{X} \times Y \longrightarrow T^{*} X
$$

the associated canonical morphisms of vector bundles.

We recall that a conic involutive submanifold $V$ of the cotangent bundle $T^{*} Z$ of a manifold $Z$ (real or complex) is regular if the restriction $\left.\omega\right|_{V}$ of the canonical 1-form $\omega$ on $T^{*} Z$ never vanishes outside the 0 -section. Recall also that if $\left(x_{1}, \cdots, x_{n}, \xi_{1}, \cdots, \xi_{n}\right)$ are canonical symplectic coordinates on $T^{*} Z$, then $\omega(x, \xi)=$ $\sum_{i=1}^{n} \xi_{i} d x_{i}$.

Let us fix some $k \in \mathbb{N}, k \geqslant 2$. In $\mathbb{C}^{k+1}=\mathbb{C}^{k} \times \mathbb{C}$ we consider the coordinates $\left(s_{1}, \cdots, s_{k}, z\right)$. As in the previous sections we set

$$
P(s, z)=z^{k}+\sum_{h=1}^{k-1}(-1)^{h} s_{h} z^{k-h}
$$

Obviously

$$
P\left(s_{1}, \cdots, s_{k}, z\right)=0 \Longleftrightarrow s_{k}=(-1)^{k-1} \sum_{h=0}^{k-1}(-1)^{h} s_{h} z^{k-h},
$$

where $s_{0}=1$. We note $s=\left(s_{1}, \cdots, s_{k}\right)$ and $s^{\prime}:=\left(s_{1}, \cdots, s_{k-1}\right)$.

Let $H$ be the smooth hypersurface of $\mathbb{C}^{k+1}$ given by the zeros of $P(s, z)$.

Let us denote by $\mathcal{L}$ the $\mathcal{D}_{\mathbb{C}^{k+1}}$-module with one generator $u$ defined by the equations $\partial u / \partial s_{1}=\cdots=\partial u / \partial s_{k}=0$. Such module is an example of a so called partial de Rham systems, which have the feature, among others, that their characteristic varieties are non singular regular involutive. In our case we have

$$
\text { Char } \mathcal{L}=\left\{(s, z) ;(\eta, \tau) \in \mathbb{C}^{k+1} \times \mathbb{C}^{k+1} \text { such that } \eta=0\right\} .
$$


Since $H \subset \mathbb{C}^{k+1}$ is defined by the equation

$$
P(s, z)=(-1)^{k} s_{k}+\sum_{h=0}^{k-1}(-1)^{h} s_{h} z^{k-h}=0,
$$

$T_{H}^{*} \mathbb{C}^{k+1}$ is the subbundle of $\left.T^{*} \mathbb{C}^{k+1}\right|_{H}$ described by

$$
\{(s, z ; \eta, \tau),(s, z) \in H, \exists \lambda \in \mathbb{C} \quad \text { such that }(\eta, \tau)=\lambda d P(s, z)\} .
$$

This means that $P(s, z)=0$ and that their exists $\lambda \in \mathbb{C}$ with $\eta_{h}=\lambda .(-1)^{h} . z^{k-h}$ for each $h \in[1, k]$ and that $\tau=\lambda \cdot P^{\prime}(s, z)$. Hence as $\eta_{k}=\lambda(-1)^{k}$ and this implies:

$$
\text { Char } \mathcal{L} \cap T_{H}^{*} \mathbb{C}^{k+1} \subset T_{\mathbb{C}^{k+1}}^{*} \mathbb{C}^{k+1}
$$

(as usual, $T_{\mathbb{C}^{k+1}}^{*} \mathbb{C}^{k+1}$ denotes the zero section of $T^{*} \mathbb{C}^{k+1}$ ), in other words $H$ is non characteristic for $\mathcal{L}$. Let us denote by $j: H \hookrightarrow \mathbb{C}^{k+1}$ the closed embedding. By Kashiwara's classical results (which can be found in [5]) it follows that the induced system $D j^{*} \mathcal{L}$ by $\mathcal{L}$ on $H$ is concentrated in degree zero and $\mathcal{N}:=\mathcal{H}^{0} D j^{*} \mathcal{L}$ is a $\mathcal{D}_{\mathbb{C}_{H}}$-coherent module whose characteristic variety is exactly

$$
j_{d} j_{\pi}^{-1} \operatorname{Char}(\mathcal{L}) .
$$

Recall that

$$
D j^{*} \mathcal{L}:=\mathcal{O}_{H} \stackrel{L}{\otimes}_{j^{-1} \mathcal{O}_{\mathbb{C}^{k+1}}} j^{-1} \mathcal{L}
$$

and in this non-characteristic case

$$
\simeq j^{-1} \frac{\mathcal{O}_{\mathbb{C}^{k+1}}}{P\left(s^{\prime}, s_{k}, z\right) \mathcal{O}_{\mathbb{C}^{k+1}}} \otimes_{j^{-1} \mathcal{O}_{\mathbb{C}^{k+1}}} j^{-1} \mathcal{L}
$$

We have

$$
\mathcal{N}:=j^{-1}\left(\frac{\mathcal{D}_{\mathbb{C}^{k+1}}}{P \mathcal{D}_{\mathbb{C}^{k+1}}+\mathcal{D}_{\mathbb{C}^{k+1}} \partial_{s_{1}}+\cdots+\mathcal{D}_{\mathbb{C}^{k+1}} \partial_{s_{k}}}\right) \simeq j^{-1}\left(\mathcal{O}_{\mathbb{C}^{k+1}} / P \mathcal{O}_{\mathbb{C}^{k+1}}\right)<\partial_{z}>
$$

which is isomorphic as a $\mathcal{D}_{H}$-module to

$$
\mathcal{O}_{H}<\partial_{z}>\simeq \frac{\mathcal{D}_{H}}{\mathcal{D}_{H} \partial_{s_{1}}+\cdots+\mathcal{D}_{H} \partial s_{k-1}}
$$

where $\partial_{s_{i}}$ stands for the derivation $\partial / \partial s_{i}$ on $\mathcal{O}_{H}$ and $\partial_{z}$ as a derivation on $\mathcal{O}_{H}$ is the class of $\partial_{z}$ in the quotient above.

In particular $\mathcal{N}$ is sub-holonomic and it is a partial de Rham system similarly to $\mathcal{L}$. Let us now determine the image of $\mathcal{N}$ under the morphism $\pi: \mathbb{C}^{k} \simeq H \rightarrow \mathbb{C}^{k}$ given by $\left(s^{\prime}, z\right) \mapsto\left(s^{\prime}, s_{k}\left(s^{\prime}, z\right)\right)$. Clearly $\pi$ is proper surjective with finite fibers.

Recall that one denotes by $\mathcal{D}_{\mathbb{C}^{k} \leftarrow H}$ the transfer $\left(\pi^{-1} \mathcal{D}_{\mathbb{C}^{k}}, \mathcal{D}_{H}\right)$-bimodule

$$
\left(\pi^{-1} \mathcal{D}_{\mathbb{C}^{k}} \otimes_{\pi^{-1} \mathcal{O}_{\mathbb{C}^{k}}} \pi^{-1} \Omega_{\mathbb{C}^{k}}^{\otimes^{-1}}\right) \otimes_{\pi^{-1} \mathcal{O}_{\mathbb{C}^{k}}} \Omega_{H}
$$

Recall also that, according to the properness and the fiber finiteness of $\pi$, we have

$$
D \pi_{*} \mathcal{N} \simeq \mathcal{H}^{0} D \pi_{*} \mathcal{N}=\pi_{*}\left(\mathcal{D}_{\mathbb{C}^{k} \leftarrow H} \otimes_{\mathcal{D}_{H}} \mathcal{M}\right)
$$

where we abusively use the notation $\pi_{*}$ for the direct image functor in the categoy of $\mathcal{D}$-modules in the two left terms and for the direct image functor for sheaves in the 
right term. According to [5, Th. 4.25 and 4.27] (see also the comments in loc.cit. before Theorem 4.27), one knows that $D \pi_{*} \mathcal{N}$ is concentrated in degree zero and that

$$
\text { Char } \mathcal{H}^{0} D \pi_{*} \mathcal{N}=\pi_{\pi} \pi_{d}^{-1} \text { Char } \mathcal{N} .
$$

So we may henceforward denote for short $\pi_{*} \mathcal{N}:=D \pi_{*} \mathcal{N}$ without ambiguity.

Let $\Delta$ as above be the zero set of the discriminant of $P$, which can also be defined as the image by $\pi$ of the subset of $H$ defined by $\left\{P^{\prime}(s, z)=0\right\}$.

Since $\pi_{d}$ is given by the $k \times k$ matrix

$$
\left(\begin{array}{cc}
I d & 0 \\
\partial s_{k} / \partial s^{\prime} & \partial s_{k} / \partial z
\end{array}\right)^{T}
$$

we conclude that Char $\pi_{*} \mathcal{N}$ is the image by $\pi_{\pi}$ of the set

$$
\left\{\left(s^{\prime}, z\right) ;\left(\eta^{\prime}, \tau\right) \in \mathbb{C}^{k} \times \mathbb{C}^{k} / \eta_{j}=-(-z)^{k-j} \cdot \tau \quad \forall j \in[1, k-1]\right\}
$$

so it is given by the set

$\left\{(s, \eta) \in \mathbb{C}^{k} \times \mathbb{C}^{k} / \exists z \in \mathbb{C}\right.$ such that $\left.P_{s}(z]\right)=0$ and with $\left.\eta_{j}=(-z)^{k-j} \eta_{k} \quad \forall j \in[1, k-1]\right\}$

Then Char $\pi_{*} \mathcal{N}$ is an involutif analytic subset of $T^{*} \mathbb{C}^{k}$ with codimension $k-1$, hence $\pi_{*} \mathcal{N}$ is a subholonomic $\mathcal{D}_{\mathbb{C}^{k+1}}$-module. Denoting by $p$ the projection: $T^{*} \mathbb{C}^{k} \rightarrow$ $\mathbb{C}^{k}$, for any $s$ such that $\Delta(s) \neq 0$, the Implicit Function Theorem implies that $p^{-1}(s) \cap$ Char $\pi_{*} \mathcal{N}$ is a cone of $l$ distinct complex lines $l_{j}=\mathbb{C}\left(\begin{array}{c}\left(-z_{j}\right)^{k-1} \\ \left(-z_{j}\right)^{k-2} \\ \cdots \\ \left(-z_{j}\right) \\ 1\end{array}\right)$, where $z_{j}$ runs over the set of $l$ distinct roots of $P(s, z)$

Proposition 4.1. The $\mathcal{D}_{\mathbb{C}^{k}}-$ module $\pi_{*} \mathcal{N}$ is the quotient of $\mathcal{D}_{\mathbb{C}^{k}}^{k} \simeq \mathcal{D}_{\mathbb{C}^{k}} \otimes_{\mathbb{C}} \mathbb{C}^{k}$ by the action of

$$
\mathcal{A}_{h}:=\partial_{s_{h}} \otimes I d_{\mathbb{C}^{k}}+(-1)^{k-h-1} \partial_{s_{k}} \otimes A(s)^{k-p} \quad \text { for } j \in[1, k-1] .
$$

Moreover the action of $z$ and $\partial_{z}$ on $\pi_{*} \mathcal{N}$ deduced from the action of $\mathcal{D}_{H}$ on $\mathcal{N}^{2}$ are given respectively by

$$
\mathcal{A}_{0}:=1 \otimes A(s) \quad \text { and } \quad \mathcal{B}:=1 \otimes \nabla+(-1)^{k-1} \partial_{s_{k}} \otimes P_{s}^{\prime}(A(s))
$$

where we put $P_{s}^{\prime}(z):=\left(\partial_{z}\left(P_{s}(z)\right)\right.$ and $\nabla:=\left(\begin{array}{cccc}0 & 0 & \ldots & 0 \\ 1 & 0 & \ldots & 0 \\ 0 & 2 & 0 & \ldots \\ \ldots & \ldots & \ldots & \ldots \\ 0 & \ldots & k-1 & 0\end{array}\right)$

\footnotetext{
${ }^{2}$ Note that $z$ and $\partial_{z}$ commute with $\partial_{s_{h}}$ for $h \in[1, k-1]$ in $\mathcal{D}_{H}$.
} 
Proof. Our goal is to explicit $\pi_{*} \mathcal{N}$ and to check that it coincides with the $\mathcal{D}_{\mathbb{C}^{k}}$ module associated to the system (@) in Theorem 2.b.1.

In a first step we explicit the transfer-module

$$
\mathcal{D}_{\mathbb{C}^{k} \leftarrow H}:=\pi^{-1} \mathcal{D}_{\mathbb{C}^{k}} \otimes_{\pi^{-1} \mathcal{O}_{\mathbb{C}^{k}}}\left(\pi^{-1} \Omega_{\mathbb{C}^{k}}^{\otimes^{-1}} \otimes_{\pi^{-1} \mathcal{O}_{\mathbb{C}^{k}}} \Omega_{H}\right)
$$

as a $\left(\pi^{-1} \mathcal{D}_{\mathbb{C}^{k}}, \mathcal{D}_{H}\right)$-bimodule.

The next step is to determine the cokernel of

$$
\begin{gathered}
\alpha:\left(\mathcal{D}_{\mathbb{C}^{k} \leftarrow H}\right)^{k-1} \longrightarrow \mathcal{D}_{\mathbb{C}^{k} \leftarrow H} \\
\left(u_{1}, \cdots, u_{k-1}\right) \mapsto \sum_{i=1}^{k-1} u_{i} \partial_{s_{i}}
\end{gathered}
$$

The last step is to apply $\pi_{*}$.

Let us denote for short

$$
\sigma:=\omega_{H} \otimes \omega_{\mathbb{C}^{k}}^{\otimes^{-1}}:=d s_{1} \wedge \cdots \wedge d s_{k-1} \wedge d_{z} \otimes\left(d s_{1} \wedge \cdots \wedge d s_{k-1} \wedge d s_{k}\right)^{\otimes^{-1}}
$$

the generator of the line bundle $\pi^{-1} \Omega_{\mathbb{C}^{k}}^{\otimes^{-1}} \otimes_{\pi^{-1} \mathcal{O}_{\mathbb{C}^{k}}} \Omega_{H}$.

Recall that $\mathcal{O}_{H}=\mathcal{O}_{\mathbb{C}^{k+1}} / \mathcal{J}$, where $\mathcal{O}$ is the ideal generated by $P(s, z)$. Hence $\mathcal{O}_{H}$ is a $\pi^{-1} \mathcal{O}_{\mathbb{C}^{k}}$-free module with rank $k$ since each section $a\left(s^{\prime}, z\right)$ of $\mathcal{O}_{H}$ is equivalent, by Weierstrass Division Theorem, to a unique polynomial $\sum_{j=0}^{k-1} a_{j}\left(s^{\prime}, s_{k}\right) z^{j}$ modulo $P(s, z)$, for some sections $a_{j}$ of $\mathcal{O}_{\mathbb{C}^{k}}$.

Hence $\mathcal{D}_{\mathbb{C}^{k} \leftarrow H}$ is a left $\pi^{-1} \mathcal{D}_{\mathbb{C}^{k}}$-free module of rank $k$ generated by the $k$-sections $\left(1 \otimes z^{j} \sigma\right)_{j=0, \cdots, k-1}$. Since the right action of each operator in $\mathcal{D}_{H}$ is $\pi^{-1} \mathcal{D}_{\mathbb{C}^{k}}$-linear, it is sufficient to calculate each $\left(1 \otimes z^{j} \sigma\right) \partial_{s_{i}}, i=1, \cdots, k-1, j=0, \cdots, k-1$.

Now recall that $H$ is defined in $\mathbb{C}^{k+1}$ by the equation $s_{k}=(-1)^{k-1} \sum_{h=0}^{k-1}(-1)^{h} s_{h} z^{k-h}$ with the convention $s_{0}=1$ and so $s_{1}, \ldots, s_{k-1}, z$ are global coordinates on $H$. Then we have in $H$

$$
\frac{\partial s_{k}}{\partial s_{h}}=(-1)^{k-h-1} z^{k-h} \text { and } \quad \frac{\partial s_{k}}{\partial z}=(-1)^{k-1} P_{s}^{\prime}(z)
$$

where $P_{s}^{\prime}(z)$ does not depend on $s_{k}$.

Let $F:=1 \otimes E(z) \sigma$ denote the basis $\left(1 \otimes z^{j} \sigma\right), j \in[0, k-1]$ of the free rank $k$ left $\pi^{-1}\left(\mathcal{D}_{\mathbb{C}^{k}}\right)$-module $\mathcal{D}_{\mathbb{C}^{k} \leftarrow H}$. Recall that, according to [5, Rem. 4.18], in view of the generators described above, the action of $\mathcal{D}_{H}$ in $\mathcal{D}_{\mathbb{C}^{k} \leftarrow H}$ is defined by the following formulas, where we consider $F$ as a $k$-vector and use the usual matrix product

$$
\begin{aligned}
& F \theta\left(s^{\prime}\right)=\theta\left(s^{\prime}\right) F \quad \text { where } \quad \theta \in \mathcal{O}_{H} \quad \text { does not depend on } z \\
& F z=A(s) F \\
& -F \partial_{s_{h}}=\partial_{s_{h}} F+(-1)^{k-h-1} \partial_{s_{k}}\left(A(s)^{k-h} F\right) \quad \forall h \in[1, k-1] \\
& -F \partial_{z}=\nabla F+(-1)^{k-1} \partial_{s_{k}}\left(P_{s}^{\prime}(A(s)) F\right)
\end{aligned}
$$

where we have used the equalities $z E(z)=A(s) E(z)$ and $\partial_{z}(E(z))=\nabla E(z)$.

Summing up: 
- For $g \in \mathcal{O}_{H}$ represented by $\sum_{r=0}^{k-1} g_{r}(s) z^{r}$, the $(k \times k)$ matrix $G$ of the $\pi^{-1} \mathcal{D}_{\mathbb{C}^{k}}$-linear morphism defined by $g$ on $\mathcal{D}_{\mathbb{C}^{k} \leftarrow H}$ is given by $G:=\sum_{r=0}^{k-1} g_{r}(s) A(s)^{r}$

- Let us consider the $\mathcal{D}_{\mathbb{C}^{k}}$-linear morphism $\alpha:\left(\mathcal{D}_{\mathbb{C}^{k}}^{k}\right)^{(k-1)} \rightarrow \mathcal{D}_{\mathbb{C}^{k}}^{k}$ defined by the following $k-1(k, k)$-matrices

$$
\mathcal{A}_{h}:=\partial_{s_{h}} \otimes I d_{\mathbb{C}^{k}}+(-1)^{k-h-1} \partial_{s_{k}} \otimes A(s)^{k-h}
$$

Let $\Phi$ be in $\mathcal{O}_{\mathbb{C}^{k}}^{k}$. In view of the relation (2), the map $\left(1 \otimes z^{h} \sigma\right) \mapsto \Phi_{h}$, for $h \in[0, k-1]$ will induce an element of $\mathcal{H} o m_{D_{\mathbb{C}^{k}}}\left(\pi_{*} \mathcal{N}, \mathcal{O}_{\mathbb{C}^{k}}\right)$, that is to say a solution of $\pi_{*} \mathcal{N}$, if and only if we have $\partial_{s_{h}}(\Phi)=(-1)^{k-h} \partial_{s_{k}}\left(A^{k-h} \Phi\right)$, since the generator of $\pi_{*} \mathcal{N}$ is anihilated by the action of $\partial_{s_{h}}$ for each $h \in[1, k-1]$.

In conclusion:

$$
\pi_{*} \mathcal{N} \simeq \operatorname{coker} \alpha
$$

by the finitness of the fibers of $\pi$.

Remark 4.2. $\mathcal{N}$ is naturally endowed with a structure of right $\Gamma\left(\mathbb{C} ; \mathcal{D}_{\mathbb{C}}\right)$-module. By functoriality $\pi_{*} \mathcal{N}$ is also a right $\Gamma\left(\mathbb{C} ; \mathcal{D}_{\mathbb{C}}\right)$-module and its structure coincides with the induced by the right $\mathcal{D}_{\mathbb{C}}$ action defined by (1) and (3) on $\mathcal{D}_{\mathbb{C}^{k} \leftarrow H} \otimes_{\mathcal{D}_{H}} \mathcal{N}$, since it commutes with each $\partial_{s_{i}}$, for $i=1, \cdots, k-1$. Therefore we obtain a natural left action of $\Gamma\left(\mathbb{C}, \mathcal{D}_{\mathbb{C}}\right)$ on $\mathcal{H}_{o} m_{\mathcal{D}_{\mathbb{C}}}\left(\pi_{*} \mathcal{N}, \mathcal{O}_{\mathbb{C}^{k}}\right)$.

We also conclude, according to Lemma 3.1 .

Proposition 4.3. The left action of $\Gamma\left(\mathbb{C} ; \mathcal{D}_{\mathbb{C}}\right)$ defined by the above Remark 4.2 on $\mathcal{H}_{\text {om }} \overline{\mathcal{D}}_{\mathbb{C}^{k}}\left(\pi_{*} \mathcal{N}, \mathcal{O}_{\mathbb{C}^{k}}\right)$ coincides with the left action of $\Gamma\left(\mathbb{C} ; \mathcal{D}_{\mathbb{C}}\right)$ on Lisbon integrals.

If $\Phi$ is a solution of $\pi_{*} \mathcal{N}$, replacing in the formula (3) the second term thanks to the equality obtained for $\phi$ after applying (2), we also derive a right action of $\partial_{z}$ which is given by the formula

$$
-\Phi \partial_{z}=\nabla \Phi+\sum_{h=0}^{k-1}(k-h) s_{h} \partial_{h+1} \Phi
$$

Our next goal is to conclude in Proposition 4.5 below that there are no global holomorphic solutions of $\pi_{*} \mathcal{N}$ other than those of the form $\Phi_{f}$, for some holomorphic function $f$ only depending on $z$. Since $j$ is non-characteristic we have an isomorphism

$$
j^{-1} R \mathcal{H} \operatorname{fom}_{\mathcal{D}_{\mathbb{C}^{k+1}}}\left(\mathcal{L}, \mathcal{O}_{\mathbb{C}^{k+1}}\right) \simeq R \mathcal{H o m}_{\mathcal{D}_{H}}\left(\mathcal{N}, \mathcal{O}_{H}\right)
$$

According to Theorem 4.33 (2) of [5], making $X=H, Y=\mathbb{C}^{k}, f=\pi, \mathcal{N}=\mathcal{O}_{\mathbb{C}^{k}}$ in loc.cit, we obtain

Theorem 4.4. For any open subset $\Omega$ of $\mathbb{C}^{k}$ we have an isomorphism functorial on $\mathcal{N}$ compatible with restrictions to open subsets

$$
R \Gamma\left(\pi^{-1}(\Omega) ; R \mathcal{H} \mathcal{H o m}_{\mathcal{D}_{H}}\left(\mathcal{N}, \mathcal{O}_{H}\right)\right) \simeq R \Gamma\left(\Omega ; R \mathcal{H} \operatorname{Hom}_{\mathcal{D}_{\mathbb{C}^{k}}}\left(\pi_{*} \mathcal{N}, \mathcal{O}_{\mathbb{C}^{k}}\right)\right)
$$


Recall that this isomorphism uses as a tool the "trace morphism": $\pi_{*} \mathcal{O}_{H} \rightarrow \mathcal{O}_{\mathbb{C}^{k}}$ constructed in [5, Prop. 4.34].

Since for any open subset $\Omega$ and any $\mathcal{D}_{H}$-module $\mathcal{P}, \Gamma(\Omega ; \cdot)$ and $\mathcal{H}$ om $_{\mathcal{D}_{H}}(\mathcal{P}, \cdot)$ are left exact functors, since if $\Omega$ is a Stein open set and if $\mathcal{P}$ admits a global resolution by free $\mathcal{D}_{H}$-modules of finite rank, then $R \mathcal{H} \operatorname{Lom}_{\mathcal{D}_{H}}(\mathcal{P}, \mathcal{O})$ is represented by a complex in degrees $\geqslant 0$ with $\Gamma(\Omega, \cdot)$-acyclic entries, we conclude that

$$
\begin{gathered}
H^{0}\left(R \Gamma\left(H ; R \mathcal{H} \operatorname{Hom}_{\mathcal{D}_{H}}\left(\mathcal{N}, \mathcal{O}_{H}\right)\right)\right)=\Gamma\left(H ; \mathcal{H} \operatorname{som}_{\mathcal{D}_{H}}\left(\mathcal{N}, \mathcal{O}_{H}\right)\right) \quad \text { and } \\
H^{0}\left(R \Gamma\left(\mathbb{C}^{k} ; R \mathcal{H} \operatorname{Fom}_{\mathcal{D}_{\mathbb{C}^{k}}}\left(\pi_{*} \mathcal{N}, \mathcal{O}_{\mathbb{C}^{k}}\right)\right)\right)=\Gamma\left(\mathbb{C}^{k} ; \mathcal{H} \operatorname{om}_{\mathcal{D}_{\mathbb{C}^{k}}}\left(\pi_{*} \mathcal{N}, \mathcal{O}_{\mathbb{C}^{k}}\right)\right)
\end{gathered}
$$

therefore Theorem 4.4 entails a $\mathbb{C}$-linear isomorphism

$$
T: \operatorname{Hom}_{\mathcal{D}_{H}}\left(\mathcal{N}, \mathcal{O}_{H}\right) \simeq \operatorname{Hom}_{\mathcal{D}_{\mathbb{C}^{k}}}\left(\pi_{*} \mathcal{N}, \mathcal{O}_{\mathbb{C}^{k}}\right)
$$

Proposition 4.5. The correspondence

$$
f \mapsto \Phi(f):=\Phi_{f}
$$

defines a $\mathbb{C}$-linear isomorphism

$$
\Phi: \Gamma\left(\mathbb{C} ; \mathcal{O}_{\mathbb{C}}\right) \longrightarrow \Gamma\left(\mathbb{C}^{k} ; \mathcal{H} \mathrm{Hom}_{\mathcal{D}_{\mathbb{C}^{k}}}\left(\pi_{*} \mathcal{N}, \mathcal{O}_{\mathbb{C}^{k}}\right)\right)=\operatorname{Hom}_{\mathcal{D}_{\mathbb{C}^{k}}}\left(\pi_{*} \mathcal{N}, \mathcal{O}_{\mathbb{C}^{k}}\right)
$$

Moreover, this isomorphism is also $\Gamma\left(\mathbb{C}, \mathcal{D}_{\mathbb{C}}\right)$-left linear.

Proof. The last statement is clear from Proposition 4.3 and Lemma 3.1 .

The remaining of the statement is equivalent to prove that $\Phi: f \mapsto \Phi_{f}$ defines an isomorphism $\operatorname{Hom}_{\mathcal{D}_{H}}\left(\mathcal{N}, \mathcal{O}_{H}\right) \rightarrow \operatorname{Hom}_{\mathcal{D}_{\mathbb{C}^{k}}}\left(\pi_{*} \mathcal{N}, \mathcal{O}_{\mathbb{C}^{k}}\right)$.

We already know that $\Phi$ is injective. It remains to prove that $\Phi$ is surjective. For

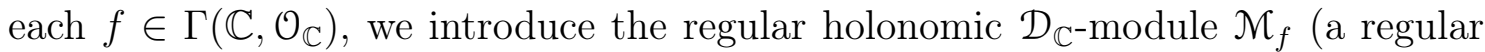
flat holomorphic connection on $\mathbb{C}$ ) of which the constant sheaf $\mathbb{C} f$ in degree zero is the complex of holomorphic solutions. We denote by $\mathcal{N}_{f}$ the regular holonomic $\mathcal{D}_{H}$-module (a regular flat holomorphic connection on $H$ )

$$
\mathcal{N}_{f}:=\mathcal{N} \otimes \mathcal{M}_{f}
$$

It is clear that $\mathcal{N}_{f}$ is a quotient of $\mathcal{N}$, and, by the left exactness of $\pi_{*}, \pi_{*} \mathcal{N}_{f}$ is a quotient of $\pi_{*} \mathcal{N}$. Moreover, according to Proposition 4.3 and Lemma 3.1, $\Phi_{f}$ belongs to $\operatorname{Hom}_{\mathcal{D}_{\mathbb{C}}}\left(\pi_{*} \mathcal{N}_{f}, \mathcal{O}_{\mathbb{C}^{k}}\right)$. According to Theorem 4.4 for each $f$ we have a $\mathbb{C}$-linear isomorphism $T_{f}: \operatorname{Hom}_{\mathcal{D}_{H}}\left(\mathcal{N}_{f}, \mathcal{O}_{H}\right) \simeq \operatorname{Hom}_{\mathcal{D}_{\mathbb{C}^{k}}}\left(\pi_{*} \mathcal{N}_{f}, \mathcal{O}_{\mathbb{C}^{k}}\right)$ which makes this last one a one dimensional $\mathbb{C}$-vector space. Moreover, by left exactness of Hom and the exactness of $\pi_{*}$, we have monomorphisms $\operatorname{Hom}_{\mathcal{D}_{\mathbb{C}^{k}}}\left(\pi_{*} \mathcal{N}_{f}, \mathcal{O}_{\mathbb{C}^{k}}\right) \subset \operatorname{Hom}_{\mathcal{D}_{\mathbb{C}^{k}}}\left(\pi_{*} \mathcal{N}, \mathcal{O}_{\mathbb{C}^{k}}\right)$ and, by functoriality, we have $T(f)=T_{f}(f)$.

We shall use the following result:

Lemma 4.6. Suppose that $f \neq 0$. Then $\operatorname{Hom}_{\mathcal{D}_{\mathbb{C}^{k}}}\left(\pi_{*} \mathcal{N}_{f}, \mathcal{O}_{\mathbb{C}^{k}}\right)$ is a one dimensional $\mathbb{C}$-vector space generated by $\Phi_{f}$. 
ProOF. The result follows by Proposition 2.3 since $\Phi_{f}$ is a non zero element of $\operatorname{Hom}_{\mathcal{D}_{\mathbb{C}^{k}}}\left(\pi_{*} \mathcal{N}_{f}, \mathcal{O}_{\mathbb{C}^{k}}\right)$ hence it is a generator as a $\mathbb{C}$-vector space.

Let us now end the proof of Proposition 4.5. Clearly $\operatorname{Hom}_{\mathcal{D}_{H}}\left(\mathcal{N}, \mathcal{O}_{H}\right)=$ $\sum_{f} \operatorname{Hom}_{\mathcal{D}_{H}}\left(\mathcal{N}_{f}, \mathcal{O}_{H}\right)$ and, according to Lemma 4.6, for each $f, \operatorname{Hom}_{\mathcal{D}_{\mathbb{C}}}\left(\pi_{*} \mathcal{N}_{f}, \mathcal{O}_{\mathbb{C}^{k}}\right)$ is the $\mathbb{C}$-vector space spanned by $\Phi_{f}$; hence $T(f)=\lambda \Phi_{f}$ for some $\lambda \in \mathbb{C}^{*}$. Since $\phi_{\lambda f}=\lambda \Phi_{f}$ we conclude that $\Phi$ is surjective which gives the desired result.

As a consequence, isomorphism $\Phi$ explicits isomorphism of Theorem 4.4 since they coincide up to the multiplication by a constant $\lambda \neq 0$.

4.a. An example. To conclude this article, let us give an interesting example of choice of the entire function $f$ on $\mathbb{C}$ for which we explicit the regular holonomic system on $\mathbb{C}^{k}$ associated to the corresponding Lisbon integrals.

The CASE $f_{t}(z):=e^{t z}$. Let us fix a parameter $t \in \mathbb{C}^{*}$ and consider the entire function $f_{t}(z):=e^{t z}$.

First remark that according to Lemma 3.1, we have $\frac{\partial E(z)}{\partial z}=\nabla E(z)$ where $\nabla$ is the $(k, k)$ matrix given by

$$
\nabla:=\left(\begin{array}{cccc}
0 & 0 & \ldots & 0 \\
1 & 0 & \ldots & 0 \\
0 & 2 & 0 & \ldots \\
\ldots & \ldots & \ldots & \ldots \\
0 & \ldots & k-1 & 0
\end{array}\right)
$$

Integration by parts in the formula $]^{3}$

$$
\Phi_{f_{t}}(s):=\frac{1}{2 i \pi} \int_{|\zeta|=R} \frac{e^{t \zeta} \cdot E(\zeta) d \zeta}{P_{s}(\zeta)}
$$

gives, using formula $(7-b i s)$ :

$$
\begin{aligned}
& t \Phi_{f_{t}}(s)=-\frac{1}{2 i \pi} \int_{|\zeta|=R} e^{t \zeta} \frac{\partial}{\partial \zeta}\left[\frac{E(\zeta)}{P_{s}(\zeta)}\right] d \zeta \\
& =-\frac{1}{2 i \pi} \int_{|\zeta|=R} e^{t \zeta} \frac{\nabla E(\zeta) P_{s}(\zeta)-P_{s}^{\prime}(\zeta) E(\zeta)}{P_{s}^{2}(\zeta)} d \zeta \\
& =-\nabla \Phi_{t}(s)+\frac{1}{2 i \pi} \int_{|\zeta|=R} e^{t \zeta} \frac{P_{s}^{\prime}(\zeta) E(\zeta)}{P_{s}^{2}(\zeta)} d \zeta \\
& (t I d+\nabla) \Phi_{f_{t}}(s)=P_{s}^{\prime}(A) \frac{1}{2 i \pi} \int_{|\zeta|=R} e^{t \zeta} \frac{E(\zeta)}{P_{s}^{2}(\zeta)} d \zeta+(-1)^{k} \frac{\partial P_{s}^{\prime}(A)}{\partial s_{k}} \Phi_{f_{t}}(s) \\
& =P_{s}^{\prime}(A)(-1)^{k-1} \frac{\partial \Phi_{f_{t}}}{\partial s_{k}}(s)+(-1)^{k-1} \frac{\partial P_{s}^{\prime}(A)}{\partial s_{k}} \Phi_{f_{t}}(s) \\
& =(-1)^{k-1} \frac{\partial\left(P_{s}^{\prime}(A) \Phi_{f_{t}}\right)}{\partial s_{k}}(s)
\end{aligned}
$$

\footnotetext{
${ }^{3}$ Remember that $t$ is a fixed non zero parameter
} 
This implies the following equation

$$
(t . I d+\nabla) P_{s}^{\prime}(A)^{-1} \Psi_{f_{t}}(s)=(-1)^{k-1} \frac{\partial\left(\Psi_{f_{t}}\right)}{\partial s_{k}}(s)
$$

for

$$
\Psi_{f_{t}}(s)=P_{s}^{\prime}(A) \Phi_{f_{t}}(s)=\frac{1}{2 i \pi} \int_{|\zeta|=R} \frac{e^{t \zeta} P_{s}^{\prime}(\zeta) E(\zeta) d \zeta}{P_{s}(\zeta)} .
$$

Combining with the system (@@) it is easy to see that we obtain a meromorphic integrable connexion on the trivial vector bundle of rank $k$ on $\mathbb{C}^{k}$ with a pole along the discriminant hypersurface $\{\Delta(s)=0\}$. The regularity of this meromorphic connexion is then consequence of the regularity of the $\mathcal{D}_{H}-$ module $\mathcal{N}_{e^{t z}}$ which is given by the equations

$$
\partial_{s_{h}}=0, \quad \forall h \in[1, k-1] \quad \text { and } \quad \partial_{z}-t=0
$$

which is clearly regular locally on $H$. So its direct image by $\pi$ (as a $\mathcal{D}_{\mathbb{C}^{k}}$-module) is regular holonomic on $\mathbb{C}^{k}$ (see [6, Th. 8.1]). 


\section{REFERENCES}

1. D. Barlet On partial differential operators annihilating trace functions, to appear.

2. D. Barlet and J. Magnússon Cycles analytiques complexes I: théorèmes de préparation des cycles, Cours Spécialisés 22, Société Mathématique de France (2014).

3. J-E Bjork Analytical D-Modules and Applications, Mathematics and Its Applications, Klwer Academic Publishers, 247 (1993).

4. M. Kashiwara, On the holonomic systems of linear differential equations II, Invent. Math., 49 , (1978) 121-135.

5. M. Kashiwara, D-modules and microlocal calculus, Translations of Mathematical Monographs, 217 American Math. Soc. (2003).

6. M. Kashiwara, The Riemann-Hilbert problem for holonomic systems, Publ. RIMS, Kyoto University, 437, (1983).

7. M. Kashiwara and P. Schapira, Sheaves on Manifolds, Grundlehren der Math. Wiss. 292 Springer-Verlag (1990).

8. M. Kashiwara and P. Schapira Moderate and formal cohomology associated with constructible sheaves, Mémoires de la SMF, 64, Société Mathématique de France (1996).

The research of T.Monteiro Fernandes was supported by Fundação para a Ciência e a Tecnologia, UID/MAT/04561/2019.

Daniel Barlet, Institut Elie Cartan, Géométrie, Université de Lorraine, Nancy, France

CNRS UMR 7502 and Institut Universitaire de France

email: daniel.barlet@univ-lorraine.fr

Teresa Monteiro Fernandes

Centro de Matemática e Aplicações Fundamentais-CIO

and

Departamento de Matemática da Faculdade de Ciências da Universidade de Lisboa, Bloco C6, Piso 2, Campo Grande, 1749-016, Lisboa Portugal

email : mtfernandes@fc.ul.pt 